\title{
Effects of modality on the neural correlates of encoding processes supporting recollection and familiarity
}

\author{
Lauren J. Gottlieb ${ }^{1,2}$ and Michael D. Rugg ${ }^{3,4,5}$ \\ ${ }^{1}$ Center for the Neurobiology of Learning and Memory, University of California at Irvine, Irvine, California 92697, USA; ${ }^{2}$ Department \\ of Neurobiology and Behavior, University of California at Irvine, Irvine, California 92697, USA; ${ }^{3}$ Center for Vital Longevity, University \\ of Texas at Dallas, Dallas, Texas 75235, USA; ${ }^{4}$ School of Behavioral and Brain Sciences, University of Texas at Dallas, Dallas,
} Texas 75235, USA

\begin{abstract}
Prior research has demonstrated that the neural correlates of successful encoding ("subsequent memory effects") partially overlap with neural regions selectively engaged by the on-line demands of the study task. The primary goal of the present experiment was to determine whether this overlap is associated solely with encoding processes supporting later recollection, or whether overlapping subsequent memory and study condition effects are also evident when later memory is familiaritybased. Subjects $(N=17)$ underwent $\mathrm{fMRI}$ scanning while studying a series of visually and auditorily presented words. Memory for the words was subsequently tested with a modified Remember/Know procedure. Auditorily selective subsequent familiarity effects were evident in bilateral temporal regions that also responded preferentially to auditory items. Although other interpretations are possible, these findings suggest that overlap between study condition-selective subsequent memory effects and regions selectively sensitive to study demands is not uniquely associated with later recollection. In addition, modality-independent subsequent memory effects were identified in several cortical regions. In every case, the effects were greatest for later recollected items, and smaller for items later recognized on the basis of familiarity. The implications of this quantitative dissociation for dual-process models of recognition memory are discussed.
\end{abstract}

Since the advent of event-related fMRI, a large literature has developed in which the neural correlates of successful memory encoding have been investigated with the "subsequent memory procedure" (for reviews, see Paller and Wagner 2002; Kim 2010). As exemplified by the meta-analysis reported by Kim (2010), a number of cortical regions have consistently been reported to demonstrate greater encoding-related activity for study items remembered on a later memory test than for forgotten items (subsequent memory effects). The cortical loci of these effects vary according to both study material (e.g., verbal vs. pictorial) and the nature of the memory test (e.g., item vs. "associative"), consistent with the idea that encoding does not depend on a single, "dedicated" cortical region or network (Rugg et al. 2002).

A number of studies have directly contrasted subsequent memory effects according to the nature of the study material (Kirchhoff et al. 2000; Reber et al. 2002; Powell et al. 2005; Uncapher et al. 2006; Uncapher and Rugg 2009; Gottlieb et al. 2010) or study task (Baker et al. 2001; Otten and Rugg 2001a; Otten et al. 2001, 2002; Fletcher et al. 2003; Mitchell et al. 2004; Park et al. 2008). Consistent with results of the above-mentioned meta-analysis (Kim 2010), most of these studies reported that the cortical loci of subsequent memory effects varied according to the characteristics of the study items or task. Importantly, in some of these studies (Otten and Rugg 2001a; Otten et al. 2002; Fletcher et al. 2003; Mitchell et al. 2004; Park et al. 2008; Gottlieb et al. 2010) cortical regions demonstrating material- and task-selective subsequent memory effects were reported to overlap with regions preferentially engaged by the corresponding study condition. In our own recent study, for example (Gottlieb et al. 2010), subsequent memory effects predictive of later memory for visual or auditory contextual information overlapped with the cortical

${ }^{5}$ Corresponding author.

E-mail mrugg@utdallas.edu.

Article is online at http://www.learnmem.org/cgi/doi/10.1101//m.2197211. regions differentially activated by visual and auditory study trials, respectively. Findings such as these have been interpreted as support for the long-standing proposal that episodic memory is a "by-product" of the on-line processing accorded a stimulus event (e.g., James 1890; Craik and Lockhart 1972; Kolers 1973; Moscovitch 1992). According to this proposal, the information encoded about an event is, in effect, a record of the processing that was engaged as it was experienced (see also Rugg et al. 2008).

With the exception of the three studies discussed below, the subsequent memory contrasts employed in the foregoing studies (all of which employed some form of recognition memory test to assess later memory) did not segregate later remembered items according to whether recognition was based on retrieval of qualitative information about the study episode (recollection), or on an acontextual sense of familiarity. There is considerable evidence that recollection and familiarity depend upon qualitatively different types of memory signal (Mandler 1980; Jacoby and Dallas 1981; Yonelinas et al. 1996; Wixted and Mickes 2010; for reviews, see Rugg and Yonelinas 2003; Yonelinas et al. 2010; see also Donaldson 1996; Dunn 2004; Rotello and Macmillan 2006 for an opposing view). The question therefore arises whether material- and task-selective subsequent memory effects reflect encoding processes that support recollection, familiarity, or both forms of memory.

As just mentioned, three studies have employed subsequent memory contrasts that permitted investigation of the effects of a study manipulation on the neural correlates of encoding processes supporting recollection. Uncapher et al. (2006) and Uncapher and Rugg (2009) had subjects study words that could appear in one of four colors and in one of four locations. The subsequent memory test required subjects to signal the color and location at study of each test item judged "old." Using as a baseline study activity elicited by words that were later recognized, but for which both color and location judgments were inaccurate, both studies reported that subsequent memory effects predictive 
of correct location and color judgments dissociated, in each case overlapping cortical regions previously implicated in the processing of the relevant feature.

Park et al. (2008) also presented study items in one of two different locations, but in this case the study manipulation was one of study task (semantic vs. syllable judgments) (cf. Otten and Rugg 2001a). The requirement in the later retrieval test was to retrieve the study location of each item endorsed as "old." The neural correlates of recollection-related encoding were operationalized in the contrast between study activity elicited by later recognized items according to whether the associated location judgment was accurate or inaccurate. Park et al. (2008) reported a double dissociation between cortical regions demonstrating recollectionrelated subsequent memory effects for the two study tasks.

Together, the findings from the above studies suggest that the localization of recollection-related subsequent memory effects varies according to study conditions. The findings do not, however, speak to the question of whether subsequent memory effects associated with later familiarity-driven recognition also vary according to study conditions. A number of scenarios can be envisaged. For example, the encoding processes that support recollection may also benefit later familiarity-based recognition. Under this scenario, study condition-specific subsequent memory effects would be evident for later recognized items regardless of whether the items were judged old on the basis of recollection or familiarity. Alternatively, and arguably more in keeping with the dual-process framework described above, the cortical loci supporting later recollection and familiarity may be anatomically distinct. Lastly, it is of course possible that study manipulations will have no impact on subsequent familiarity effects.

The present study was designed to permit subsequent memory effects elicited by study items to be directly contrasted according to their modality of presentation (visual vs. auditory), thereby allowing identification of modality-dependent subsequent memory effects (cf. Gottlieb et al. 2010). We employed a memory task (a modified "Remember/Know" procedure) (Yonelinas et al. 2005) that permitted subsequently recognized items to be segregated according to whether they were associated with recollection or familiarity as operationalized by subjective report. Subjects studied a series of words presented either visually or auditorily. The test task required one of five responses to be made to each test item. If subjects recollected a contextual detail associated with a test item, they endorsed the item as "Remembered." In the absence of recollection, the requirement was to judge whether the item was old or new, using a four-point confidence scale that ranged from "confident old" to "confident new." The advantage of this procedure is that it minimizes (though it does not eliminate-see Discussion) the confound that would otherwise exist between memory "strength" and the distinction between recollection and familiarity (Wixted et al. 2010). Using this approach, we were able to address the question whether "subsequent familiarity effects" overlap with modality-selective regions.

\section{Results}

\section{Behavioral performance}

\section{Study task}

Mean accuracy on the study task was $0.95(\mathrm{SD}=0.02)$ and 0.89 $(\mathrm{SD}=0.05)$ for visual items and auditory items, respectively. These values differed significantly $\left(t_{(16)}=6.08, P<0.001\right)$.

Reaction times (RTs) to the study items are shown in Table 1, segregated according to later memory performance. Mean RT for judgments on visually presented words was $1145 \mathrm{msec}(\mathrm{SD}=$ 211), compared with $1518 \mathrm{msec}(\mathrm{SD}=229)$ for auditory words. To parallel the fMRI analyses described below, RT analyses were
Table 1. Mean reaction times ( $\mathrm{msec}$ ) for correct size decisions at study segregated by subsequent memory

\begin{tabular}{lccc} 
& $\mathbf{R}$ & $\mathbf{4}$ & $\mathbf{3 2 1}$ \\
\hline Visual & $1185(142)$ & $1117(137)$ & $1070(127)$ \\
Auditory & $1514(186)$ & $1455(138)$ & $1566(207)$ \\
\hline
\end{tabular}

conducted for three subsequent memory conditions, " $\mathrm{R}$ " (recollection), " 4 " (familiarity), and "3/2/1" (miss). A $2 \times 3$ ANOVA (factors of modality [visual and auditory] and later memory [R, 4 , and $3 / 2 / 1])$ revealed main effects of modality $\left(F_{(1,16)}=\right.$ $228.29, P<0.001)$ and subsequent memory $\left(F_{(1.7,27.9)}=4.74\right.$, $P<0.05)$, along with a modality $\times$ subsequent memory condition interaction $\left(F_{(1.7,27.8)}=13.27, P<0.001\right)$. In the visual condition, RTs for items that went on to be recollected were significantly slower than items that were later judged familiar $\left(t_{(16)}=2.71, P<0.05\right)$, and RTs for items that were subsequently judged familiar did not significantly differ from items that were subsequently forgotten. In the auditory condition, in contrast, RTs for items that were either later recollected or judged as familiar did not significantly differ, whereas RTs for words that were later missed were significantly slower than RTs for items that went on to be judged familiar $\left(t_{(16)}=3.87, P<0.01\right)$.

\section{Retrieval task}

Accuracy data for old and new items can be found in Figure 1A (visual modality) and Figure 1B (auditory modality). Hit rate collapsed over " $\mathrm{R}$, " " 4 ," and " 3 " responses was $0.86(\mathrm{SD}=0.05)$ against a false alarm rate of $0.16(\mathrm{SD}=0.13)$ for visually presented items. For auditory items, hit and false alarm rates were $0.74(\mathrm{SD}=$ $0.06)$ and $0.16(\mathrm{SD}=0.07)$, respectively. Recognition performance, indexed by the discrimination metric of hit rate minus false alarm rate, was $0.71(\mathrm{SD}=0.12)$ and $0.58(\mathrm{SD}=0.08)$ for visual and auditory items, respectively $\left(t_{(16)}=3.58, P<0.01\right)$. As Figure 1 suggests, the proportions of old and new items receiving a " 3 " response did not significantly differ in either the visual or the auditory condition. Thus the memory signal available when subjects made an "unconfident old" response did not reliably discriminate between old and new items. Therefore, for the purposes of the fMRI analyses described below, items receiving " 3 ," "2," and " 1 " responses were collapsed to form a generic "miss" condition. ${ }^{6}$

We performed an additional analysis on " $\mathrm{R}$ " and " 4 " responses using a metric that permitted memory "strength" in these response categories to be directly compared (see Wixted et al. 2010). Accuracy in each category was defined as the proportion of hits divided by the sum of hits and false alarms. This index was calculated separately for visual " $\mathrm{R}$," visual " 4 ," auditory " $\mathrm{R}$," and auditory " 4 " conditions. The resulting values were 0.99 , $0.89,0.97$, and 0.91 , respectively. A $2 \times 2$ ANOVA on these data revealed a main effect of response category $\left(F_{(1,16)}=16.34 ; P<\right.$ $0.001)$, indicating greater strength for $\mathrm{R}$ than for 4 responses, but no effect of modality or its interaction with response category.

\section{fMRI results}

We first identified regions demonstrating subsequent memory effects common to the two modality conditions (modality

${ }^{6}$ To determine whether the inclusion of items receiving an "unconfident old" judgment in the "miss" category biased the resulting subsequent memory effects, we performed an additional analysis in which these items were excluded. Albeit at a reduced statistical threshold (a consequence of reduced trial numbers and the need to exclude one subject with too few missed trials), this additional analysis yielded results essentially identical to those obtained in the original analysis. 


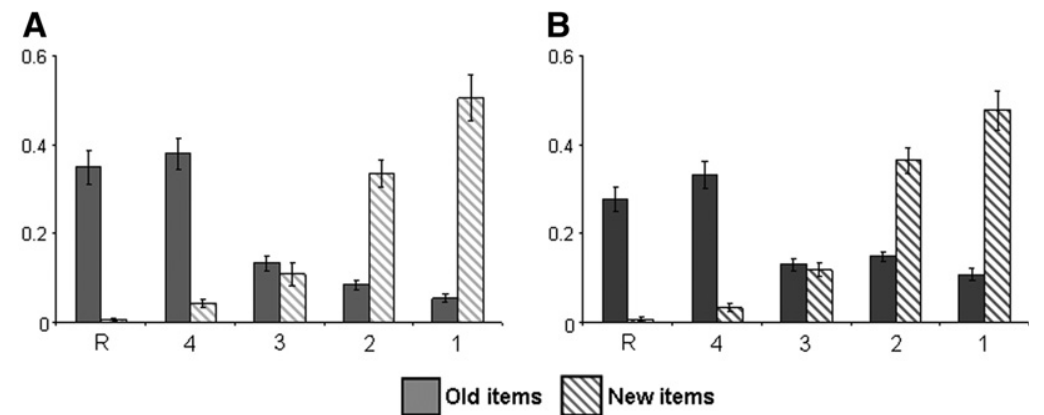

Figure 1. Performance on the subsequent memory test. (A) Visual, $(B)$ auditory.

Recollect $>$ Familiar and the Familiar $>$ Miss contrasts were reliable in both the visual and auditory conditions ( $\max$ 1-tailed $P<0.05)$.

\section{Modality-selective subsequent memory effects that overlap generic modality effects}

We predicted that modality-selective subsequent memory effects would overlap the respective main effect of modality, as in Gottlieb et al. (2010). We performed hypothesis-driven analyses to test this prediction by employing pairwise contrasts derived from the ANOVA

nonselective effects). We then performed analyses to assess whether, as in Gottlieb et al. (2010), modality-selective subsequent memory effects (subsequent memory effects that were reliable for items presented in only one of the two modalities) overlapped regions demonstrating generic modality effects.

\section{Modality nonselective subsequent memory effects}

Regions demonstrating subsequent memory effects were identified in an unbiased manner using a $2 \times 3$ ANOVA design (factors of modality [visual, auditory] and subsequent memory [R, 4, 3/ 2/1]). To identify regions demonstrating modality-independent subsequent memory effects, the SPM of the main effect of subsequent memory (thresholded at $P<0.001$ ) was exclusively masked by the modality $\times$ memory interaction (thresholded at $P<0.1$ ), thus removing all voxels where effects significantly differed (at $P<0.05$, one-sided) according to modality of the study items. As detailed in Table 2 and illustrated in Figure 2, these modality nonselective effects were localized to left intra-parietal sulcus (IPS), bilateral fusiform cortex, left parahippocampal cortex/hippocampus, left middle frontal gyrus, left inferior frontal gyrus (IFG), and left lateral orbital gyrus.

To investigate whether any of these effects were preferentially associated with recollection or familiarity, encoding-related activity in each significant cluster, characterized as the mean parameter estimate of the peak voxel in the cluster, was collapsed across modality and entered into a $3 \times 7$ ANOVA (factors of subsequent memory [R, 4, 3/2/1] and region [left IPS, left fusiform cortex, right fusiform cortex, left parahippocampal cortex/ hippocampus, left middle frontal gyrus, left IFG, left lateral orbital gyrus]; see Fig. 2). This analysis revealed main effects of region $\left(F_{(3.2,50.4)}=9.64, P<0.001\right)$ and subsequent memory $\left(F_{(1.4,22.4)}=23.55, P<0.001\right)$. There was, however, no evidence of a subsequent memory $\times$ region interaction $\left(F_{(6.0,95.9)}=1.21\right.$, $P=0.31$ ). To further characterize the nature of these effects, two additional $2 \times 7$ ANOVAs were performed to directly compare the parameter estimates associated with the specific subsequent memory conditions; namely, recollection vs. familiar and familiar vs. miss. These analyses revealed a stepwise function across the three subsequent memory conditions, such that parameter estimates associated with recollection were significantly greater than those associated with familiarity (main effect of subsequent memory, $\left.F_{(1,16)}=8.98, P<0.01\right)$, and the parameter estimates associated with familiarity were significantly greater than those associated with subsequent misses (main effect of subsequent memory, $F_{(1,16)}=35.40, P<0.001$ ) (see Fig. 2). These follow up ANOVAs also failed to detect any subsequent memory $x$ region interactions (minimum $P=0.13$ ). Finally, we used pairwise contrasts on the data collapsed across region to assess whether this stepwise pattern for the response categories was evident for each modality separately. This was the case: Both the model. Subsequent recollection effects for visually presented study items that overlapped the corresponding modality effect were identified by inclusively masking the visual recollection contrast $(\mathrm{R}>4, P<0.01)$ with the main effect of modality (visual $>$ auditory; $P<0.001$ ), giving a conjoint significance level across the two contrasts of $P<0.0001$ (Fisher 1950; Lazar et al. 2002). To ensure that any resulting effects were selective for the visual modality, surviving voxels were exclusively masked with the auditory recollection contrast (thresholded at $P<0.05$ ). The analogous procedure was used to identify regions demonstrating an auditorily selective subsequent recollection effect. The same approach was also used to identify regions demonstrating modality-selective subsequent familiarity effects $(4>321)$.

Visual effects. A single cluster in right fusiform cortex (xyz $=51,-54$, $-15 ; Z=3.42 ; 26$ voxels) demonstrated a subsequent visual recollection effect that overlapped regions preferentially engaged during the processing of visual items. This finding should be treated with circumspection, however. The locus of the effect overlaps the right fusiform region previously identified as demonstrating a modality non-selective effect, that is, a region where there was no overall response category by modality interaction (Table 2; Fig. 2). Moreover, analysis of the peak parameter estimates revealed a robust auditory subsequent memory effects for both " $\mathrm{R}$ " and "confident old" items relative to misses, indicating that subsequent memory effects were not modality-selective. We therefore think it likely that the seemingly differential patterning of the recollection effects between the two modalities in this region may be a Type I error and requires replication before strong interpretation. No regions could be identified that exhibited a visually selective familiarity effect.

Auditory effects. No voxels exhibited a subsequent recollection effect $(\mathrm{R}>4)$ within auditorily selective regions. However, robust subsequent familiarity effects $(4>321)$ were identified in bilateral superior temporal sulcus (see Table 3; Fig. 3).

Table 2. Regions demonstrating modality-independent subsequent memory effects

\begin{tabular}{|c|c|c|}
\hline Coordinates $(x, y, z)$ & Z (\# voxels) & Region \\
\hline $\begin{array}{l}-30,-69,42 \\
-51,-48,-21 \\
-39,-27,-18\end{array}$ & $\begin{array}{l}3.89(67) \\
5.03(181) \\
5.04\end{array}$ & $\begin{array}{l}\text { L intra-parietal sulcus } \\
\text { Left fusiform cortex } \\
\text { L hippocampus/ } \\
\text { parahippocampal cortex }{ }^{\text {a }}\end{array}$ \\
\hline $\begin{array}{l}-27,9,51 \\
-45,18,15 \\
-39,39,-18 \\
51,-48,-18\end{array}$ & $\begin{array}{l}4.26(106) \\
4.81(189) \\
4.17(40) \\
4.89(44)\end{array}$ & $\begin{array}{l}L \text { middle frontal gyrus } \\
L \text { posterior inferior frontal gyrus } \\
L \text { lateral orbital gyrus } \\
R \text { fusiform cortex }\end{array}$ \\
\hline
\end{tabular}

${ }^{a}$ Cluster contiguous with left fusiform cortex. 

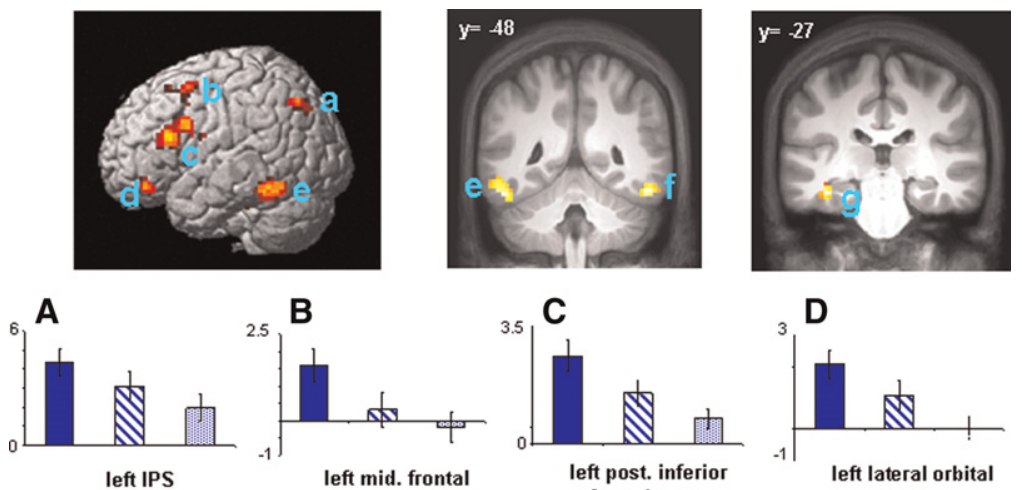

left IPS left mid. frontal gyrus

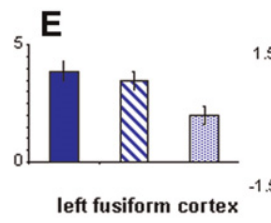

$\mathbf{F}$ frontal gyrus

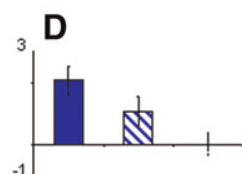

left lateral orbital gyrus

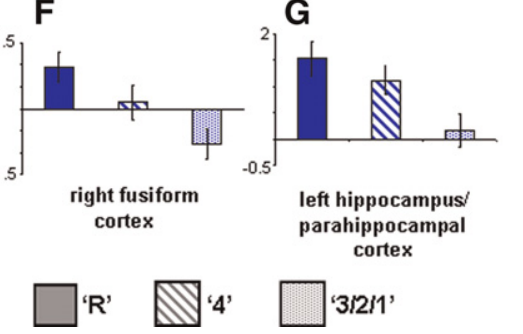

Figure 2. (Upper) Modality-independent subsequent memory effects (main effect of memory $[P<$ $0.001]$ exclusively masked with subsequent memory by modality interaction $[P<0.1]$ ). Effects are rendered onto a single subject template brain (left) and projected onto a section of the normalized average anatomical image (right). (Lower) Bar plots show parameter estimates (in arbitrary units) for recollected (R), familiar (4), and forgotten (3/2/1) trials of peak voxels for effects localized in (A) left IPS $(-30,-69$, $42),(E, F)$ left $(-51,-48,-21)$ and right fusiform cortex $(51,-48,-18),(B)$ left middle frontal gyrus $(-27,9,51),(C)$ left posterior inferior frontal gyrus $(-45,18,15),(D)$ left lateral orbital gyrus $(-39,39$, $-18)$, and $(G)$ left hippocampus/parahippocampal cortex $(-39,-27,-18)$.

\section{Modality-selective subsequent memory effects unconstrained by generic modality effects}

We also searched for modality-selective subsequent recollection and familiarity effects independently of whether the effects overlapped with generic modality effects. Pairwise contrasts derived from the ANOVA model were again employed. Subsequent visual recollection effects were identified by exclusively masking the visual recollection contrast $(\mathrm{R}>4$, thresholded at $P<0.001)$ with the visual familiarity contrast $(4>321$, thresholded at $P<0.05)$ in order to remove voxels associated with subsequent familiarity. The surviving voxels were then exclusively masked with the recollection contrast in the auditory modality (thresholded at $P<0.05$ ) to ensure that resulting effects were unique to the visual condition. The same procedure was used to identify subsequent auditory recollection effects. An analogous approach was used to identify subsequent visually and auditorily selective familiarity effects.

Visual effects. Recollection effects selective for the visual modality are summarized in Table 4 and illustrated in Figure 4 . The effects were localized to right superior frontal gyrus, right anterior cingulate cortex, left superior frontal sulcus, and left insula. No regions were identified that were associated with later familiarity.

Auditory effects. No regions exhibiting auditorily selective subsequent recollection effects could be identified. However, subsequent familiarity effects were identified in bilateral middle occipital gyrus, right inferior occipital gyrus, and posterior perirhinal cortex (see Table 4; Fig. 5).

\section{Confounding effects of differences in study RTs}

As described in the behavioral results, study RTs for visually presented items were significantly slower when the items went on or judged familiar. We first identified regions where subsequent memory effects were common to the two classes of study item. None of these regions demonstrated subsequent memory effects that strongly dissociated items according to whether they were later recollected or recognized on the basis of familiarity only. Instead, study activity in these regions exhibited a graded profile across subsequent memory conditions (i.e., recollect $>$ familiar $>$ miss). Additionally, we identified subsequent memory effects that were reliable for one modality but not the other. These modality-selective effects dissociated according to recollection and familiarity, in that we identified subsequent recollection effects that were reliable in the visual condition only, and familiarity effects that were only reliable in the auditory condition. Replicating prior results, we also identified auditorily selective effects that overlapped cortical regions preferentially engaged during the processing of auditory study items.

\section{Behavioral findings}

Study RTs were significantly slower for items subsequently recollected than for items later forgotten in the visual condition, while the opposite trend was evident for RTs in the auditory condition for items later judged familiar relative to those that were forgotten. The reasons for these disparate subsequent memory RT effects

Table 3. Auditory subsequent familiarity effects overlapping auditorily selective regions

\begin{tabular}{lcc}
\hline Coordinates $(\mathbf{x}, \mathbf{y}, \mathbf{z})$ & $\mathbf{Z}$ (\# voxels) & Region \\
\hline$-42,-27,-12$ & $3.00(45)$ & L superior temporal sulcus \\
$54,-27,3$ & $2.90(23)$ & R superior temporal sulcus \\
$66,-6,-3$ & $3.26(31)$ & R superior temporal gyrus \\
\hline
\end{tabular}



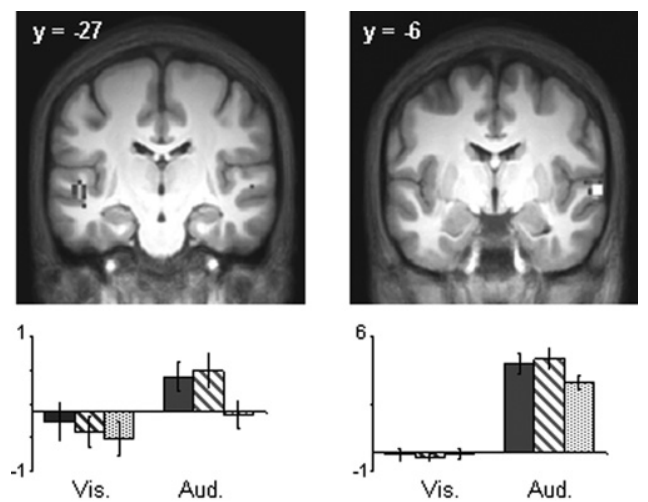

left sup. temporal sulcus

right sup. temporal gyrus

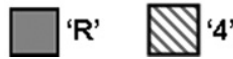

'3/2/1'

Figure 3. (Upper) Auditorily selective subsequent familiarity effects $(P<$ $0.01)$ that overlap regions demonstrating auditorily selective activity $(P<$ 0.001 ). (Lower) Bar plots of mean parameter estimates (in arbitrary units) for recollected, familiar, and forgotten study items in left superior temporal sulcus and right superior temporal gyrus.

are unclear, and little help in their interpretation is available from prior studies, where findings have been inconsistent. Thus, whereas longer RTs for later remembered relative to later forgotten items were reported in some prior studies (Wagner et al. 1998; Morcom et al. 2003; Uncapher and Rugg 2005a; Duverne et al. 2009), opposite (Otten and Rugg 2001a,b) or null findings (Otten et al. 2001; Uncapher and Rugg 2005b) were reported in others. Despite the disparate RT effects, similar patterns of fMRI subsequent memory effects were reported across these studies, making it unlikely that the fMRI effects depend upon a specific pattern of differences in study RTs. Moreover, in the present study we obtained very similar fMRI findings when analysis was restricted to subsets of trials selected so as to eliminate subsequent memory effects in RTs.

Recognition memory performance was more accurate for visually than for auditorily presented study words. This performance difference likely reflects the greater difficulty of identifying auditorily relative to visually presented words in the context of the scanner environment, in that the auditory words had to be discriminated against the background of scanner noise. Thus, more attentional resources were likely devoted to early perceptual processing in the case of the auditory items, leaving fewer resources available for the semantic/elaborative processing that is well known to benefit later explicit memory (Craik and Lockhart 1972).

As in Wixted et al. (2010), we operationalized "memory strength" for items in each response category in terms of the accuracy with which items were judged old. There were highly reliable differences in memory strength between $\mathrm{R}$ and 4 response categories but no difference in strength between modalities. The finding that items endorsed as "remembered" and "confident old" differed in memory strength raises the possibility of a confound between strength and the recollection/familiarity distinction (Wixted et al. 2010). We discuss the implications of this confound below.

\section{fMRI findings}

Using a procedure that was unbiased with respect to the identification of subsequent memory effects associated with recollection or familiarity, we identified modality nonselective effects in several regions that have been implicated in episodic encoding by numerous prior studies (for review, see Kim 2010). Among these regions were left IPS, bilateral fusiform cortex, left IFG, and left parahippocampal cortex/hippocampus. Strikingly, the subsequent memory effects in each of these regions were graded; study activity was greatest for later recollected items, intermediate in magnitude for items judged familiar, and lowest for forgotten items (see Fig. 2). Thus, in the context of the present experiment, there was no evidence that these regions supported encoding uniquely associated with either recollection or familiarity. Clearly, a parsimonious account of these findings is that encoding-related activity in these regions does not honor the distinction between recollection and familiarity, but instead imparts varying levels of memory "strength" to study items, the level of which influences whether the items will later be endorsed as recollected or familiar. Such an interpretation is in line both with some cognitive models of the "Remember/Know" distinction (e.g., Donaldson 1996; Dunn 2004; Rotello and Macmillan 2006) and with related interpretations of the relevant functional neuroimaging literature (Squire et al. 2007; Wais 2008; Wixted et al. 2010).

An alternative interpretation of these findings is possible, however. This hinges on the proposal (e.g., Wixted 2007; Wixted and Mickes 2010) that the signal supporting recollection is continuous, and not thresholded as assumed in standard dualprocess models (e.g., Yonelinas 2001). According to this alternative interpretation, subjects in the present experiment adopted a relatively conservative criterion for " $R$ " judgments, such that many of the items accorded a "confident old" judgment carried a recollection signal sufficiently strong to be detected in a subsequent memory contrast. This is not implausible in the context of the present experiment: The strength of the memory signal (as indexed by recognition accuracy) supporting recognition judgments was very high for many items (accuracy was $\geq 0.9$ for items endorsed both as recollected or confident old), yet the instructions emphasized the importance of spreading judgments across the available response categories. To comply with these instructions, it is possible that subjects used "confident old" judgments for items that, in other circumstances, would have been endorsed as recollected. This interpretation is consistent with prior evidence suggesting that encoding-related activity in some of the above regions, notably the hippocampus, parahippocampal cortex, and left IFG, selectively supports recollection (e.g., Summerfield et al. 2006; Park and Rugg 2008; Diana et al. 2010; Park and Rugg 2011).

A final interpretation of these graded subsequent memory effects-suggested by two anonymous reviewers-is that the

Table 4. Modality-selective subsequent memory effects unconstrained by global modality effects

\begin{tabular}{lcl}
\hline & Visual recollection effects \\
\hline Coordinates $(x, y, z)$ & Z (\# voxels) & \multicolumn{1}{c}{ Region } \\
\hline$-42,-3,15$ & $3.71(22)$ & L insula \\
$-18,9,42$ & $4.25(29)$ & L superior frontal sulcus \\
$3,21,18$ & $3.81(21)$ & R anterior cingulate cortex \\
$9,36,48$ & $5.25(136)$ & R superior frontal gyrus \\
\hline \multicolumn{4}{c}{ Auditory familiarity effects } \\
\hline Coordinates $(\mathbf{x}, \mathbf{y}, \mathbf{z})$ & $\mathbf{Z}$ (\# voxels) & \multicolumn{1}{c}{ Region } \\
\hline$-42,-81,24$ & $3.57(23)$ & L middle occipital gyrus \\
$-30,-21,-24$ & $3.83(28)$ & L perirhinal cortex \\
$39,-87,-6$ & $3.78(36)$ & R inferior occipital gyrus \\
$45,-75,21$ & $4.24(94)$ & R middle occipital gyrus \\
\hline
\end{tabular}



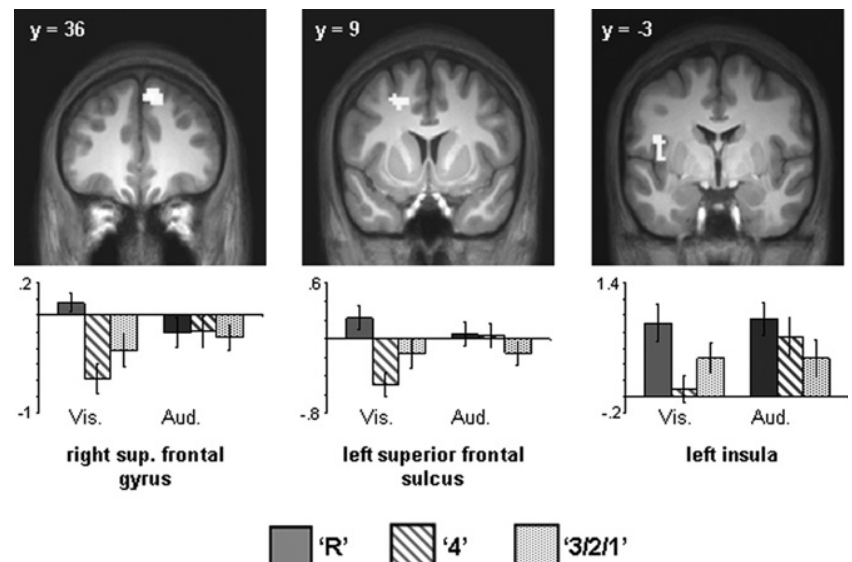

'3/2/1'

Figure 4. (Upper) Visually selective subsequent recollection effects projected onto sections of the normalized across-subjects averaged anatomical image. (Lower) Bar plots of mean parameter estimates (in arbitrary units) for recollected (R), familiar (4), and forgotten $(3 / 2 / 1)$ trials in right superior frontal gyrus, left superior frontal sulcus, and left insula.

effects reflect encoding processes that selectively support familiarity. By this argument, "R" judgments are associated not only with greater recollection than "confident old" judgments, but also with stronger familiarity. Whereas this possibility cannot be discounted on the basis of the present findings, we think that it is unlikely to suffice as an explanation of the effects in their entirety. As was just noted, subsequent memory effects in some of the regions identified here have been linked specifically with recollection in prior studies employing other memory test procedures. One of these procedures in particular, associative recognition, has long been regarded as being heavily, if not exclusively, dependent upon recollection (e.g., Mickes et al. 2010). Thus, the finding that associative subsequent memory effects are consistently reported in left inferior frontal cortex and hippocampus suggests that these effects do not reflect encoding processes that support familiarity exclusively.

\section{Modality-selective subsequent memory effects}

The analysis of modality-selective subsequent memory effects focused on the prediction that some of these effects should overlap with regions that responded preferentially to the corresponding class of study trials (cf. Gottlieb et al. 2010). This prediction was confirmed for auditory subsequent familiarity effects. Reminiscent of our prior study (Gottlieb et al. 2010), these effects were evident in lateral temporal regions that also responded preferentially to auditory relative to visual items. Crucially, these effects were no greater in magnitude for items later endorsed as recollected than they were for items later judged as highly familiar. Such a pattern of effects is usually interpreted as evidence for an effect selectively associated with familiarity, on the assumption that recollected items invariably carry a significant familiarity signal (e.g., Davachi et al. 2003; Uncapher et al. 2006). Thus the present findings shed additional light on the interpretation of the modality-selective subsequent memory effects reported by Gottlieb et al. (2010). In that study, subsequent memory effects were identified by the contrast between later recognized study items for which the modality of an associated context word was correctly recalled vs. a mixture of study items associated with inaccurate context memory or failed recognition. Hence, it was not possible to determine whether the effects were uniquely associated with recollection. The present findings suggest that, at least for auditory study items, modality-selective lateral temporal subsequent memory effects either support familiarity exclusively or, perhaps, contribute to both familiarity and recollection. More generally, the present findings suggest that subsequent memory effects localized to cortical regions selectively engaged by the on-line demands of the study task are not confined to encoding processes that support later recollection. Of course, this conclusion is predicated on the twin assumptions that items endorsed as "remembered" carried a stronger recollection signal than those endorsed "confident old," and that this signal would have been reflected in enhanced BOLD activity were these subsequent memory effects selectively associated with recollection.

In addition to identifying modality-selective subsequent memory effects that overlapped with visually or auditorily responsive cortical regions, we were also able to identify modalityselective effects in other cortical regions. Strikingly, these effects were uniformly associated with recollection in the case of the visual items, and with familiarity in the case of the auditory modality. The reasons for this dissociation are unclear. One possibility is that it reflects greater across-trial variability in the setting of the response criterion for "remember" judgments in the auditory than in the visual condition, leading to a weaker segregation between the remember and confident old response categories according to the "strength" of the underlying memory signal. ${ }^{7}$

This possibility notwithstanding, the auditorily selective subsequent familiarity effect identified in left posterior perirhinal cortex deserves mention. The effect was equal in magnitude for items associated with later recollection or familiarity, a finding consistent with several prior studies in which the perirhinal cortex demonstrated familiarity-selective encoding-related activity (Davachi et al. 2003; Ranganath et al. 2004; Staresina and Davachi 2006, 2008; Uncapher et al. 2006; but see Squire et al. 2007). It is unclear, however, why the perirhinal effect in the present study was confined to auditory items only (even at a liberal threshold of 0.05, we were unable to detect perirhinal effects for visual items).

In conclusion, the present study questioned whether it is possible to identify modality-selective subsequent familiarity effects that overlap with corresponding modality-selective regions. The answer to this question is a qualified yes. Subsequent familiarity effects demonstrated a single dissociation, with modality-selective effects evident for auditorily but not visually presented words. Moreover, a subset of these effects overlapped with regions that were auditorily selective. These findings suggest that modality-selective subsequent memory effects are not uniquely associated with encoding processes that support later recollection.

\section{Materials and Methods}

\section{Subjects}

Twenty-five subjects consented to participate in the study. All reported themselves to be in good general health, right-handed, to have no history of neurological disease or contra-indications for MR imaging, and to have attained fluency in English by age five. They were recruited from the University of California, Irvine (UCI) community, and remunerated for their participation, in accordance with the human subjects procedures approved by the Institutional Review Board of UCI. The data from eight subjects were excluded from all analyses: Two subjects had $<10$ trials in the visual "remember" response category (N's of 2 and 3, respectively), three subjects had $<10$ trials in the auditory "confident old" category (N's of 7, 1, and 4, respectively), one subject had more false alarms than correct rejections in the visual condition,

${ }^{7}$ We thank an anonymous reviewer for bringing this possibility to our attention. 


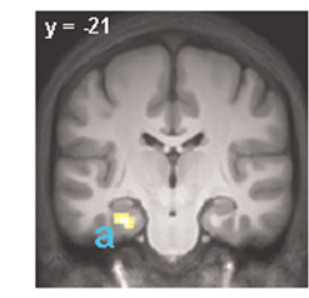

A

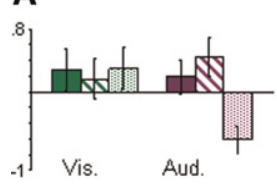

left perirhinal cortex

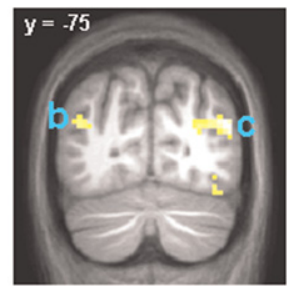

B

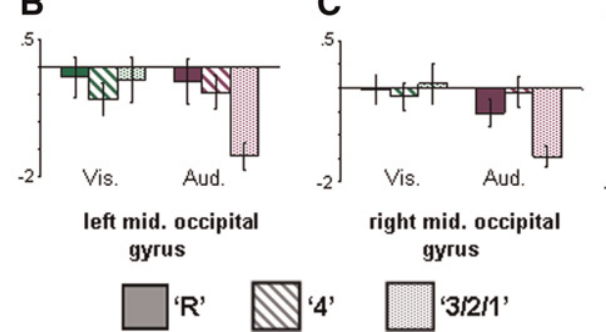

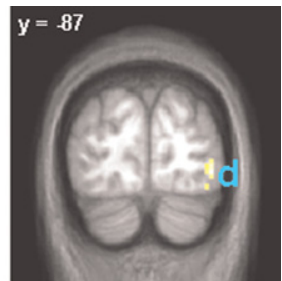

D

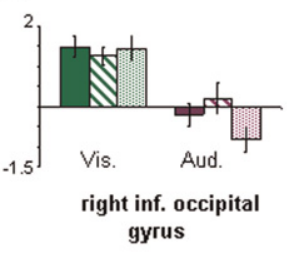

Figure 5. (Upper) Auditorily selective subsequent familiarity effects projected onto sections of the normalized across-subjects averaged anatomical image. (Lower) Bar plots of mean parameter estimates (in arbitrary units) for recollected (R), familiar (4), and forgotten (3/2/1) trials in (A) left perirhinal cortex, $(B)$ left middle occipital gyrus, $(C)$ right middle occipital gyrus, and $(D)$ right inferior occipital gyrus.

and one subject failed to recognize more than half of the old auditory stimuli at test. Data from a final subject was excluded because of excessive motion during scanning $(>4 \mathrm{~mm})$. Data are reported from the remaining 17 subjects (11 males), who ranged in age from 18 yr to $29 \mathrm{yr}$ (mean, $21 \mathrm{yr}$ ).

\section{Stimulus materials}

Experimental stimuli were drawn from a pool of 433 concrete words, obtained from the MRC Psycholinguistic Database (http:// www.psy.uwa.edu.au/mrcdatabase/uwa_mrc.htm). Each word was formatted for presentation in visual and auditory modalities. The words ranged in length from three to nine letters (mean length six) and had a Kucera-Francis written frequency ranging between one and 275 occurrences per million (mean frequency of 21). Approximately half of the words denoted objects bigger than a shoebox and the other half denoted objects that were smaller than a shoebox. Visually presented words were displayed in black uppercase 30 point Helvetica font on a gray background. Auditory words were recorded in a male voice, edited to a constant sound pressure level and filtered to remove ambient noise (http:// audacity.sourcefourge.net). Auditory stimuli were presented binaurally via MR compatible headphones and did not exceed $1000 \mathrm{msec}$ in duration (mean duration $650 \mathrm{msec}$ ). Of the 433 words, 12 were selected to be used for practice study and test lists, and 10 were selected to be used as buffers. Of the remaining 411 words, 266 were selected on a subject-specific basis to serve as critical stimuli. For each study block, 200 words were selected to serve as critical study stimuli, of which 100 were randomly designated to be presented in the visual modality, while the other 100 words were presented in the auditory modality. Two study lists were created; each list contained a pseudo-random ordering of 50 words presented visually, 50 words presented auditorily, and two buffers at the beginning and end of each study list. The study task required a decision as to whether or not each word denoted an object that was bigger or smaller than a shoebox.

Test items consisted of the 200 words from the two study blocks in addition to 66 new words. Old test items were presented in the same modality as at study. Of the new items, half were presented visually and the other half auditorily. The test requirement was to judge whether each item was old or new using a four-point confidence scale plus a "remember" option. Both study and test items were presented in a subject-specific pseudo-random order, such that there were no more than three consecutive presentations of items belonging to any one experimental condition. At study, visually presented words were back-projected onto a screen binaurally via headphones with the volume adjusted to a comfortable hearing level for each subject.

\section{Experiment tasks and procedures}

The experiment comprised a single study-test cycle.

\section{Study procedure}

Instructions and practice on the study task were administered outside the scanner. The study phase of the experiment proper consisted of the presentation of two blocks of items, separated by a brief rest period ( $\sim 1 \mathrm{~min})$. Each study trial began with the presentation of a red fixation character in the center of the display frame for $500 \mathrm{msec}$. In the visual condition, the character was replaced by a visual word that was displayed for $500 \mathrm{msec}$, after which it was replaced by a centrally presented black fixation for 2500 msec, completing the visual condition trial. For auditory trials, the red fixation character was replaced by the black fixation character for $3000 \mathrm{msec}$, coinciding with the onset of the word (auditory condition). Subjects were informed they would receive no warning as to the modality of the word to be presented on each trial. Size judgments were signaled by button press of left or right index fingers, and response mapping was counterbalanced across subjects. Instructions placed equal emphasis on speed and accuracy. The stimulus onset asynchrony (SOA) of the study trials was stochastically distributed with a minimum interval of 3500 msec modulated by the addition of 66 pseudo-randomly intermixed null trials (Josephs and Henson 1999). Trials were presented in a pseudo-random order, with no more than three trials of one item-type (visual, auditory, or null) occurring consecutively. Each study block consisted of 137 trials, comprising 50 critical visual study items, 50 critical auditory items, 33 null events, and four buffer items to give a total of 200 critical study items across two blocks.

\section{Test procedure}

Following the completion of the second study block, volunteers exited the scanner and were taken to the neighboring testing room. Only then were they informed of the memory test and given instructions and a short practice test. Approximately $30 \mathrm{~min}$ elapsed between the completion of the second study block and the beginning of the memory test proper. Each test trial began with a red fixation cross presented in the center of a gray frame for $500 \mathrm{msec}$, followed by the presentation of either an auditory 
or a visual word. After word presentation, a black fixation cross was centrally presented until the subject responded, signaling the end of the trial. The test items consisted of two buffers at the onset of the test block, the 200 critical study words and 66 randomly interspersed unstudied (new) items (no more than three items of one type were presented consecutively). The new items were evenly divided between visual and auditory modalities. Subjects were instructed to make one of five responses to each test word: " $\mathrm{R}$ " = remember, $4=$ confident old, $3=$ unconfident old, $2=$ unconfident new, $1=$ confident new, and were told to spread their responses over the full range of recognition categories. The " $R$ " response was described as indicating recall of some contextual detail about the word's study episode. Examples of such contextual details were given, such as memory for a specific thought associated with the word, memory for a mental image generated in association with the word, or memory for some distinctive aspect of the study item (e.g., its visual or phonological properties). Subjects were instructed that, in the absence of such recall, they should signal their judgment whether the word was old or new using the four-point confidence scale described above. To encourage compliance with the instructions, during the practice test subjects were instructed to describe the specific contextual detail or details they retrieved in association with each " $\mathrm{R}$ " response. The test was self-paced, with instructions to complete the test as quickly as possible without sacrificing speed for accuracy. The test was presented as a single block that lasted approximately 15 min.

\section{fMRI data acquisition}

A Philips Achieva 3T MR scanner (Philips Medical Systems) was used to acquire both T1-weighted anatomical images $(240 \times 240$ matrix, $1-\mathrm{mm}^{3}$ voxels, 160 slices, sagittal acquisition, 3D MP-RAGE sequence) and T2*-weighted echoplanar images (EPI) $(80 \times 79$ matrix, $3 \times 3 \mathrm{~mm}$ in-plane resolution, axial acquisition, flip angle $70^{\circ}$, echo time [TE] $30 \mathrm{msec}$ ) optimized for blood oxygenation level dependent (BOLD) contrast. The data were acquired using a sensitivity encoding (SENSE) reduction factor of 1.5 on an eight-channel parallel imaging headcoil. Each EPI volume comprised 303 -mm-thick axial slices oriented parallel to the AC-PC plane and separated by 1-mm gaps, positioned to give full coverage of the cerebrum and most of the cerebellum. Data were acquired in two sessions of 259 volumes each, with a repetition time (TR) of $2 \mathrm{sec} /$ volume, leading to an effective sampling rate of $2 \mathrm{~Hz}$. Slices were acquired in an ascending sequential order. The first five volumes of each session were discarded to allow equilibration of tissue magnetization.

\section{fMRI data analysis}

The MRI data were preprocessed and analyzed with SPM5 (Wellcome Department of Imaging Neuroscience; http://www .fil.ion.ucl.ac.uk/spm) (Friston et al. 1995) implemented in MATLAB 7.6 (The MathWorks Inc). All functional volumes were spatially realigned initially to the first volume of the first study block and, subsequently, to the across-block mean. The data in each volume were temporally shifted to the onset of the middle slice and the resulting volumes were coregistered with the anatomical volume. Each subject's anatomical volume was segmented into gray and white matter (Ashburner and Friston 2005), and then processed with the DARTEL SPM toolbox (Ashburner 2007) to create an across-subjects $(N=17)$ template. Parameters were determined by an affine transformation of the template to Montreal Neurological Institute (MNI) space, along with the DARTEL-based transformation parameters, and were then applied to each subject's anatomical and functional data (resampled to $1-\mathrm{mm}$ and $3-\mathrm{mm}$ isotropic voxels, respectively). The normalized fMRI data were smoothed with an 8-mm FWHM Gaussian kernel. Functional time series were concatenated across sessions.

Statistical analyses were performed on the study phase data in two stages of a mixed effects model. In the first stage, neural activity elicited by the study words was modeled by delta functions (impulse events) that coincided with the onset of each word. The ensuing BOLD response was modeled by convolving the neural functions with a canonical hemodynamic response function (HRF) and its temporal and dispersion derivatives (Friston et al. 1998) to yield regressors in a General Linear Model (GLM) that modeled the BOLD response to each event-type.

For the reasons discussed in the Results section above, the principal analyses were confined to six events of interest: studied visual and auditory words that were later remembered (visual recollected hits and auditory recollected hits, respectively), visual and auditory words that were later confidently endorsed as old (visual familiar hits and auditory familiar hits, respectively), and studied words that, on the later memory test, were associated either with unconfident old, unconfident new, or confident new judgments (visual and auditory misses). A seventh and eighth category of trials comprised visual and auditory events of no interest, namely buffer trials and trials associated with incorrect or omitted study responses. Six regressors modeling concatenated movement-related variance (three rigid-body translations and three rotations determined from the realignment stage) and sessionspecific constant terms modeling the mean over scans in each session were also entered into the design matrix.

For each voxel, the functional time series was high-pass-filtered to $1 / 128 \mathrm{~Hz}$ and scaled within-session to yield a grand mean of 100 across voxels and scans. Parameter estimates for events of interest were estimated using a GLM. Nonsphericity of the error covariance was accommodated by an AR(1) model, in which the temporal autocorrelation was estimated by pooling over suprathreshold voxels (Friston et al. 2002). The parameters for each covariate and the hyperparameters governing the error covariance were estimated using restricted maximum likelihood (ReML). Parameter estimates for the six conditions of interest (recollected hits, familiar hits, and misses for the visual and auditory conditions, respectively) were derived for each participant and carried forward to a second level groupwise analysis. In this analysis, individual participants' parameter estimates for the six conditions of interest were entered into a repeated-measures twoway ANOVA with factors of modality (visual, auditory) and response category (recollect, familiar, miss). Within the ANOVA model, $F$ ratios were estimated with respect to a single pooled error term and thresholded at $P<0.001$. Unless otherwise specified, only effects surviving an uncorrected threshold of $P<0.001$ were interpreted. The peak voxels of clusters exhibiting reliable effects are reported in MNI coordinates. Protection against Type I error was effected by using the Analysis of Functional Neuroimages (AFNI) AlphaSim tool (http://afni.nimh.nih.gov/ afni/doc/manual/AlphaSim.html) to estimate the minimum cluster size necessary for a clusterwise corrected significance level of $P<0.05$ at a height-threshold of $P<0.001$. The critical value was 21 contiguous voxels.

\section{Acknowledgments}

This research was supported by the National Institute of Mental Health (NIH1R01MH074528). The authors thank the members of the UCI Research Imaging Center for their assistance with fMRI data acquisition.

\section{References}

Ashburner J. 2007. A fast diffeomorphic image registration algorithm. NeuroImage 38: 95-113.

Ashburner J, Friston KJ. 2005. Unified segmentation. NeuroImage 26: $839-851$.

Baker JT, Sanders AL, Maccotta L, Buckner RL. 2001. Neural correlates of verbal memory encoding during semantic and structural processing tasks. Neuroreport 12: 1251-1256.

Craik F, Lockhart R. 1972. Levels of processing: A framework for memory research. J Verb LearVerb Behav 11: 671-684.

Davachi L, Mitchell JP, Wagner AD. 2003. Multiple routes to memory: Distinct medial temporal lobe processes build item and source memories. Proc Natl Acad Sci 100: 2157-2162.

Diana RA, Yonelinas AP, Ranganath C. 2010. Medial temporal lobe activity during source retrieval reflects information type, not memory strength. J Cogn Neurosci 22: 1808-1818. 
Donaldson W. 1996. The role of decision processes in remembering and knowing. Mem Cognit 24: 523-533.

Dunn JC. 2004. Remember-know: A matter of confidence. Psychol Rev 111: 524-542.

Duverne S, Motamedinia S, Rugg MD. 2009. The relationship between aging, performance, and the neural correlates of successful memory encoding. Cereb Cortex 19: 733-744.

Fisher R. 1950. Statistical methods for research workers, 11th ed. Oliver \& Boyd, London.

Fletcher PC, Stephenson CME, Carpenter TA, Donovan T, Bullmorel ET. 2003. Regional brain activations predicting subsequent memory success: An event-related fMRI study of the influence of encoding tasks. Cortex 39: 1009-1026.

Friston K, Holmes AP, Worsely K, Poline J, Frith C, Frackowiak R. 1995. Statistical parametric maps in functional imaging: A general linear approach. Hum Brain Mapp 2: 189-210.

Friston KJ, Fletcher P, Josephs O, Holmes A, Rugg MD, Turner R. 1998. Event-related fMRI: Characterizing differential responses. NeuroImage 7: $30-40$.

Friston KJ, Penny W, Phillips C, Kiebel S, Hinton G, Ashburner J. 2002. Classical and Bayesian inference in neuroimaging: Theory. NeuroImage 16: $465-483$.

Gottlieb LJ, Uncapher MR, Rugg MD. 2010. Dissociation of the neural correlates of visual and auditory contextual encoding. Neuropsychologia 48: $137-144$.

Jacoby LL, Dallas M. 1981. On the relationship between autobiographical memory and perceptual learning. J Exp Psychol Gen 110: 306-340.

James W. 1890. Memory. In Principles of psychology, Vol. 1, pp. 643-696. Dover Publications, New York.

Josephs O, Henson RN. 1999. Event-related functional magnetic resonance imaging: Modelling, inference and optimization. Philos Trans R Soc Lond B Biol Sci 354: $1215-1228$.

Kim H. 2010. Neural activity that predicts subsequent memory and forgetting: A meta-analysis of 74 fMRI studies. Neurolmage 54: $2446-2461$.

Kirchhoff BA, Wagner AD, Maril A, Stern CE. 2000. Prefrontal-temporal circuitry for episodic encoding and subsequent memory. J Neurosci 20: 6173-6180.

Kolers P. 1973. Remembering operations. Mem Cognit 1: 347-355.

Lazar NA, Luna B, Sweeney JA, Eddy WF. 2002. Combining brains: A survey of methods for statistical pooling of information. NeuroImage 16: $538-550$.

Mandler G. 1980. Recognizing: The judgment of previous occurrence. Psych 87: 252-271.

Mickes L, Johnson EM, Wixted JT. 2010. Continuous recollection versus unitized familiarity in associative recognition. J Exp Psychol 36: 843-863.

Mitchell JP, Macrae CN, Banaji MR. 2004. Encoding-specific effects of social cognition on the neural correlates of subsequent memory. J Neurosci 24: $4912-4917$

Morcom AM, Good CD, Frackowiak RSJ, Rugg MD. 2003. Age effects on the neural correlates of successful memory encoding. Brain 126: 213-229.

Moscovitch M. 1992. Memory and working with memory: A component process model based on modules and central systems. J Cogn Neurosci 4: $257-267$

Otten LJ, Rugg MD. 2001a. Task-dependency of the neural correlates of episodic encoding as measured by fMRI. Cereb Cortex 11: 1150-1160.

Otten LJ, Rugg MD. 2001b. Electrophysiological correlates of memory encoding are task-dependent. Brain Res Cogn Brain Res 12: 11-18.

Otten LJ, Henson RN, Rugg MD. 2001. Depth of processing effects on neural correlates of memory encoding: Relationship between findings from across- and within-task comparisons. Brain 124: 399-412.

Otten LJ, Henson RNA, Rugg MD. 2002. State-related and item-related neural correlates of successful memory encoding. Nat Neurosci 5: $1339-1344$.

Paller KA, Wagner AD. 2002. Observing the transformation of experience into memory. Trends Cogn Sci 6: 93-102.

Park H, Rugg MD. 2008. Neural correlates of successful encoding of semantically and phonologically mediated inter-item associations. NeuroImage 43: $165-172$.

Park H, Rugg MD. 2011. Neural correlates of encoding within- and acrossdomain inter-item associations. J Cogn Neurosci 23: 2533-2543.
Park H, Uncapher MR, Rugg MD. 2008. Effects of study task on the neural correlates of source encoding. Learn Mem 15: 417-425.

Powell HWR, Koepp MJ, Symms MR, Boulby PA, Salek-Haddadi A, Thompson PJ, Duncan JS, Richardson MP. 2005. Material-specific lateralization of memory encoding in the medial temporal lobe: Blocked versus event-related design. NeuroImage 27: 231-239.

Ranganath C, Yonelinas AP, Cohen MX, Dy CJ, Tom SM, D'Esposito M. 2004. Dissociable correlates of recollection and familiarity within the medial temporal lobes. Neuropsychologia 42: 2-13.

Reber PJ, Siwiec RM, Gitelman DR, Parrish TB, Mesulam M, Paller KA, Gitleman DR. 2002. Neural correlates of successful encoding identified using functional magnetic resonance imaging. J Neurosci 22: 9541-9548.

Rotello CM, Macmillan NA. 2006. Remember-know models as decision strategies in two experimental paradigms. J Mem Lang 55: 479-494.

Rugg M, Yonelinas A. 2003. Human recognition memory: A cognitive neuroscience perspective. Trends Cogn Sci 7: 313-319.

Rugg MD, Otten LJ, Henson RNA. 2002. The neural basis of episodic memory: Evidence from functional neuroimaging. Philos Trans $R$ Soc Lond B Biol Sci 357: 1097-1110.

Rugg MD, Johnson JD, Park H, Uncapher MR. 2008. Encoding-retrieval overlap in human episodic memory: A functional neuroimaging perspective. Prog Brain Res 169: 339-352.

Squire LR, Wixted JT, Clark RE. 2007. Recognition memory and the medial temporal lobe: A new perspective. Nat Rev Neurosci 8: 872-883.

Staresina BP, Davachi L. 2006. Differential encoding mechanisms for subsequent associative recognition and free recall. J Neurosci 26: 9162-9172.

Staresina BP, Davachi L. 2008. Selective and shared contributions of the hippocampus and perirhinal cortex to episodic item and associative encoding. J Cogn Neurosci 20: 1478-1489.

Summerfield C, Greene M, Wager T, Egner T, Hirsch J, Mangels J. 2006. Neocortical connectivity during episodic memory formation. PLoS Biol 4: pe128. doi: 10.1371/journal.pbio.0040128.

Uncapher MR, Rugg MD. 2005a. Encoding and the durability of episodic memory: A functional magnetic resonance imaging study. J Neurosci 25: $7260-7267$.

Uncapher MR, Rugg MD. 2005b. Effects of divided attention on fMRI correlates of memory encoding. J Cogn Neurosci 17: 1923-1935.

Uncapher MR, Rugg MD. 2009. Selecting for memory? The influence of selective attention on the mnemonic binding of contextual information. J Neurosci 29: 8270-8279.

Uncapher MR, Otten LJ, Rugg MD. 2006. Episodic encoding is more than the sum of its parts: An fMRI investigation of multifeatural contextual encoding. Neuron 52: 547-556.

Wagner AD, Schacter DL, Rotte M, Koutstaal W, Maril A, Dale AM, Rosen BR, Buckner RL. 1998. Building memories: Remembering and forgetting of verbal experiences as predicted by brain activity. Science 281: $1188-1191$.

Wais PE. 2008. FMRI signals associated with memory strength in the medial temporal lobes: A meta-analysis. Neuropsychologia 46: 3185-3196.

Wixted JT. 2007. Dual-process theory and signal-detection theory of recognition memory. Psychol Rev 114: 152-176.

Wixted JT, Mickes L. 2010. A continuous dual-process model of remember/ know judgments. Psychol Rev 117: 1025-1054.

Wixted JT, Mickes L, Squire LR. 2010. Measuring recollection and familiarity in the medial temporal lobe. Hippocampus 20: 1195-1205.

Yonelinas AP. 2001. Components of episodic memory: The contribution of recollection and familiarity. Philos Trans R Soc Lond B Biol Sci 356: $1363-1374$.

Yonelinas AP, Dobbins I, Szymanski MD, Dhaliwal HS, King L. 1996. Signal-detection, threshold, and dual-process models of recognition memory: ROCs and conscious recollection. Conscious Cogn 5: 418-441.

Yonelinas AP, Otten LJ, Shaw KN, Rugg MD. 2005. Separating the brain regions involved in recollection and familiarity in recognition memory. J Neurosci 25: 3002-3008.

Yonelinas AP, Aly M, Wang W, Koen JD. 2010. Recollection and familiarity: Examining controversial assumptions and new directions. Hippocampus 20: 1178-1194.

Received March 4, 2011; accepted in revised form June 23, 2011. 


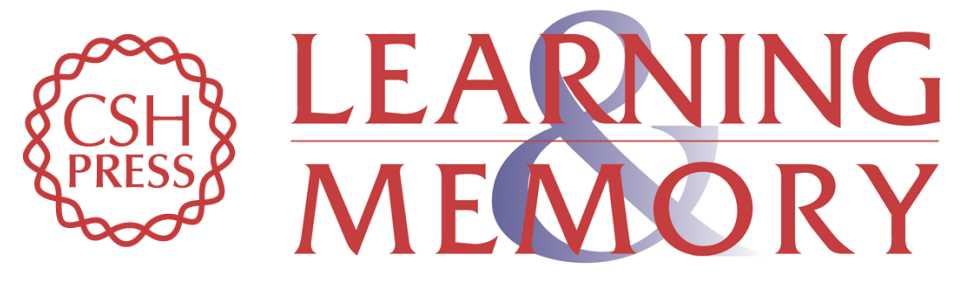

\title{
Effects of modality on the neural correlates of encoding processes supporting recollection and familiarity
}

\author{
Lauren J. Gottlieb and Michael D. Rugg
}

Learn. Mem. 2011, 18:

Access the most recent version at doi:10.1101//m.2197211

References This article cites 58 articles, 10 of which can be accessed free at:

http://learnmem.cshlp.org/content/18/9/565.full.html\#ref-list-1

License

Email Alerting Receive free email alerts when new articles cite this article - sign up in the box at the Service top right corner of the article or click here. 